\title{
DEPRESSION, NOT LETHARGY, MODERATES THE FRAILTY-SUBJECTIVE HEALTH RELATIONSHIP AMONG CENTENARIANS
}

\author{
J S K Kwan ${ }^{1}$, B H P Lau ${ }^{2}$, P Martin ${ }^{3}$, K S L Cheung ${ }^{4}$ \\ 1. Department of Medicine, The University of Hong Kong (HKU) \\ 2. Department of Psychology, HKU \\ 3. College of Human Sciences, lowa State University \\ 4. Sau Po Centre on Ageing and Department of Social Work and Social Administration, HKU
}

\section{Introduction}

Very old people may be physically frail, but they may not necessarily experience poor subjective health after taking into account other dimensions of health, e.g. psychological well-being. We hypothesized that the frailty-subjective health relationship is moderated by depression and/or lethargy in very-old adults.

\section{Methods}

We conducted a cross-sectional community-based centenarian study of 153 Chinese near-and centenarians. We assessed a) FRAIL Questionnaire of the International Academy of Nutrition and Aging (FRAIL-IANA); b) Geriatric Depression Scale-Short Form (GDS-SF); and c) Subjective Health (1=very bad to $5=$ very good). Hierarchical regression was conducted to test the moderation effect of depression, adjusting for age, gender, living arrangement, socio-economic status and cognition. We then conducted another hierarchical regression analysis using only the "lethargy" items of the GDS-SF.

\section{Results}

According to FRAIL-IANA, 20\% of the centenarians were non-frail, $56 \%$ were pre-frail, and $24 \%$ were frail. Mean scores (SD) for GDS-SF and Subjective Health were 2.6 (3.7) and 3.3 (0.9) respectively. Living with family (vs. living alone), favourable socio-economic status, and lower level of frailty were significant predictors of Subjective Health. The interaction term between frailty and depression was significant $(\beta=-$ $0.21, \mathrm{P}=0.024)$, and the overall model was significant, explaining $27.7 \%$ of the variance of Subjective Health. Inspection of the simple slopes confirmed those who were less depressed had a weaker frailtysubjective health relationship. There was no significant moderation effect with only the "lethargy" items of the GDS-SF.

\section{Conclusions}

Depression, not lethargy, moderates the frailty-subjective health relationship among centenarians. Our result therefore implies that a protective psychological mechanism may enable centenarians to maintain an optimistic view of their health despite their physical frailty. Future studies should explore the psychosocial mechanisms used by oldest-old adults to cope with their frailty and daily functional constraints. 